\title{
Bilan des recherches sur le judaïsme au Maghreb dans l'Antiquité *
}

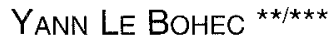

L'histoire du judaïsme dans le Maghreb des Anciens a été relativement peu étudiée. Pourtant, elle devrit intéresser au moins trois catégories de chercheurs, et en premier lieu ceux qui se sont spécialisés dans l'étude de cette nation et de cette religion. Ensuite, on ne peut pas étudier le christianisme nous y reviendrons - sans tenir compte de ce monothésime qui l'a précédé, et avec lequel il a été en concurrence. Enfin les historiens, du monde romain en général et de l'Afrique romaine en particulier, ne peuvent pas faire l'impasse sur cette communauté. M. Le Glay ${ }^{1}$, à la suite de Chr. Courtois ${ }^{2}$, avait attiré l'attention sur une problemátique à la fois essentielle et négligée, à laquelle J.$M$. Lassère ${ }^{3}$ et moimême ${ }^{4}$ nous nous sommes attachés; ces deux savants avaient en effet remarqué que tous les travaux, au XIX e et au Xxe siècles, avaient privilégié les liens qui unissaient les provinces à Rome ${ }^{5}$; ils souhaitaient attirer l'attention sur les liens qui unissaient les provinces entre elles, et les mouvements de migration présentent, de ce point de vue, le plus vif intérêt.

\footnotetext{
* Ponencia presentada en el Encuentro Internacional de Historiadores «En torno a Sefarad». Toledo 1991.

** Professeur à l'Université de Lyon III.

*** Debido que el artículo del profesor Yann Le Bohec fue impreso en el último número de esta revista con incorreciones y erratas, volvemos a incluirlo en el presente, esperando poder así subsanar tan lamentable incidente, rogando a la vez, al profesor Le Bohec nos disculpe.

LE GLAY, M., "Les Gaulois en Afrique", dans Mél. A. Grenier = Coll. Latomus, LVIII, 1962, pages $995-1029$.

2 Courtols, Chr., "Les rapports entre l'Afrique et la Gaule au début du Moyen-Age», CT II, 1954, pages $127-128$.

3 LASSĖRE, J:-M., Vbique, populus, 1977, pages 413-426.

4 LE BOHEC, Y., "Inscriptions juives et judaïsantes de l'Afrique romaine", Ant. Afr., XVII, 1981, pages 165-207, et "Juifs et judaïsants dans l'Afrique romaine. Remarques onomastiques", ibidem, pages 209-229; «Les sources archéologiques du judaïsme africain», dans Juifs et judaïsme dans l'Afrique du nord dans l'Antiquité et le Haut moyen-Age, sous la direction de $\mathrm{C}$. lancu et $\mathrm{J}$ - $-\mathrm{M}$. Lassère, 1985, pages 13-47, et en collaboration avec LE BOHEC, S., "Juifs et chrétiens de la Judée à l'Afrique", dans L'historie des croyants. Mémoire vivante des hommes. Mél. Ch. Molette, 1989, pages 567-575.

5 On pense au titre du livre devenu un classique de R. Bianchi Bandinelli, Rome le centre du pouvoir, 1969.
} 
L'examen d l'historiographie montre effectivement le peu d'attrait des milieux érudits pour les antiquités juives du Maghreb. Ici, tout avait pourtant commencé dans l'abondance. Entre 1906 et 1911, notamment, N. Slousch avait publié plusieurs ouvrages qui aboutissaient tous aux mêmes conclusions ${ }^{6}$. D'abord, la langue hébraïque était apparentée, et de près, à celle des Phéniciens. Donc, partout où vivaient des Phéniciens, vivaient des Hébreux. Il avait été suivi, para la suite, par M. Mieses ", bien qu'il fût arrivé à cette conclusion ahurissante: "La civilisation de Carthage apparaît purement et simplement hébraïque" ${ }^{8}$. II réussissait ainsi à faire le tableau d'un judaïsme africain à la fois très précoce, puisqu'il était implanté au plus tard dès le vile siècle avant J.-C., et très prolifique, puisqu'il était représenté dans toutes les échelles puniques. Hélas, toute cette belle construction doit être mise à bas. La parenté entre les deux langues n'est pas aussi étroite que l'a cru $\mathrm{N}$. Slousch. Elle n'implique pas, de toute façon, une présence automatique de Juifs là où il y avait des Phéniciens (la parenté qui existe incontestablement entre la français et l'espagnol n'implique pas la présence d'importantes communautés francophones là où sont attestées des communautés hispanophones, et vice versa, d'ailleurs). Le principal argument contre les hypothèses de $\mathrm{N}$. Slousch tient dans l'absence totale de traces archéologiques, épigraphiques ou autres, d'un judaïsme africain préromain. Nos propres recherches dans ce domaine ${ }^{9}$ ont été aussi infructueuses que celles de J.-M. Lassère ${ }^{10}$. Lors du congrès qui s'est tenu à Montpellier en 1983, M. Sznycer avait fait une courte communication à ce sujet, communication qui n'a malheureusement pas été donnée pour les actes, et qui allait dans le même sens.

Le premier travail réellement scientifique est dû à $P$. Monceaux. En 1904, il avait publié les inscriptions juives d'Afrique, mais en les considérant comme une annexe des inscriptions chrétiennes ${ }^{11}$. Par ailleurs, il avait

6 SLousch, N. Étude sur l'histoire des Juifs et le judaïsme au Maroc, 1906, 67 pages; HébréoPhéniciens et Judéo-Berbères, 1908, 473 pages; Les Hébréo-Phéniciens. Introduction à l'histoire des origines de la colonisation hébraique dans les pays méditerranéens, 1909, 206 pages; Un voyage d'études juives en Afrique, 1909, 87 pages; La civilisation hébraïque et phénicienne à Carthage, 1911, 31 pages.

7 MIESES, M., "Les Juifs et les établissements puniques en Afrique du nord", REJ, XCII, 1932 , pages 113-135; XCIII, 1933, pages 57-72 et 135-156, et XCIV, 1933, pages 73-89.

a Slousch, N., La civilisation hébraĭque et phénicienne à Carthage, 1911, page 6.

9 LE BOHEC, Y., Ant. Afr., XVII, 1981, page 201-202.

10 LASSERE, J.-M., Vbique populus, 1977, page 413.

"Monceaux, P., "Enquête sur l'épigraphie chrétienne d'Afrique; les inscriptions juives» [sic.], $R A, 1904$, I, pages 354-373, et «Païens judaïsants, essai d'interprétation d'une inscription africaine», $R A, 1902$, pages 208-226. 
donné un petit article de synthèse, qui a été réimprimé en $1971^{12}$, où il s'efforçait de rassembler tous les autres documents disponibles pour dessiner un tableau aussi complet que possible. II arrivait ainsi à proposer des listes de dix communautés, plus cinq ou six individus isolés, et sept mentions de judaïsants. De cette liste, il fallut retirer les lieux dits «Henchir Youdi», «La Ferme du Juif», qui avaient toutes les chances de remonter au Moyen Age, voir à des temps plus récents encore, plutôt qu'a l'Antiquité. Le seul apport notable à ce travail est dû à J. Ferron qui a retrouvé des épitaphes dans la nécropole de Gamart, à Carthage, grâce à des photographies prises à la lumière infrarouge ${ }^{13}$.

La rareté et la concision des sources avaient alors découragé toutes les tentatives de synthèse. Pour connaître les premiers Juifs installés au Maghreb, il convient d'utiliser des ouvrages très généraux sur le judaïsme quand ils consentent à consacrer quelques passages á cette region éloignée de la Terre Promise. Aussi ne négligera-t-on pas l'article ludaei du Dictionnaire des Antiquités de Ch. Daremberg et E. Saglio; il a été écrit par Th. Reinach ${ }^{14}$. On trouvera de même des renseignements utiles et des points de vue pleins de bon sens dans les synthèses devenues classiques d'E. Schürer ${ }^{15}$ et J. Juster ${ }^{16}$ et, en dernier lieu, dans le livre de H. Z. Hirschberg dont il existe une traduction anglaise ${ }^{17}$.

Depuis quinze ans, la question a été renouvelée par une série d'études qui s'efforçaient les unes de présenter les sources de manière critique, les autres d'esquisser des synthèses. Quatre types de documents ont été ainsi présentés à la communauté scientifique.

En ce qui concerne l'épigraphie, qu'il fallait dégager du contexte chrétien et rendre à sa spécificite judaïque, l'auteur de cette communication a proposé un nouveau corpus des "Inscriptions juives et judaïsantes de l'Afrique romaine" en $1981^{18}$. Bien que quelques documents aient été

\footnotetext{
12 Monceaux, P., «Les colonies juives dans l'Afrique romaine», $R E J$, 1904, pages 1-28 = CT, XVIII, 1970, pages 5-30.

13 FERRON, J., “Inscriptions juives de Carthage», Cahiers de Byrsa, I, 1951, pages 175-206; «Epigraphie juive», ibidem, VI, 1956, págs. 99-192, et pl. I, et «Un hypogée juif», ibidem, VI, 1956, pages 105-117.

${ }_{14}$ Reinach, Th., s.u. Iudaei, dans Ch. Daremberg et E. Saglio, Dictionnaire des Antiquités, III, 1, 1900, 624 et suiv.

15 SHürER, E., Geschichte des jüdischen Volkes im Zeitalter Jesu Christi, III, 4, 1909, 53 et suiv.

16 JUSTER, J., Les Juifs dans l'Empire romain, 1914, 207 et suiv.

17 Hirschberg, H.Z.J.W., A History of the Jews in North Africa, I, From Antiquity to the 16th Century, 1974, 2 e édit., 518, en particulier 21-86.

i8 LE BOHEC, Y, «Inscriptions juives et judaïsantes de l' Afrique romaine», Ann. $t$. Afr., XVII, $1981,165-207$.
} 
retirés de la liste de $P$. Monceaux, et grâce aux nouvelles découvertes qui ont été faites cent vingt quatre textes connaître onze communautés et dix sites où sont connus des individus isolés. Ont été ainsi regroupés des inscriptions incontestablement juives, et d'autres simplement «judaïsantes» 19. H. Solin a fait remarquer l'imprécision que recouvre ce terme ${ }^{20}$, à la suite d'autres savants il est vrai; de fait, si le mot peut avoir plusieurs sens très différents, il peut aussi recouvrir des degrés également très différents; on peut ainsi avoir un personnage plus ou moins «judaïsant» et les noms dérivés du sabbat n'impliquent pas de manière obligatoire des liens très étroits avec la religiion de Moïse. Les Juifs africains, donc, on les retrouve principalement en Afrique Proconsulaire, en particulier à Carthage, où ils ont utilisé l'importante nécropole de Gamarth, et à Hammam Lif, où a été fouillée une synagogue. Dans la même province mentionons également Utique et Hippone, les autres parties de l'Afrique semblent avis accueilli moins de communautés. On en connaît a Tripoli et à Medinet et Sultan en Tripolitaine, à Henchir Fouara en Numidie, à Sétif, Tipasa et Cherchel en Maurétanie Césarienne, et à Volubilis en Tingitane Pour en revenir au recueil d'inscriptions qui vient d'être évoqué, il faut ajouter que les notes de bas de pages donnent en prime un assez grand nombre de références bibliographiques; elles dépassent le cadre étroit de la seule épigraphie, et nous y renvoyons. Depuis la parution de cet article, F. Vattioni a cru pouvoir ajouter à cette liste un texte de Lepcis Magna ${ }^{21}$; nous restons cependant sceptique sur le caractère juif attaché a ce document qui paraît d'interpretation très difficile.

Cette recherçhe sur l'épigraphie avait montré l'importance de l'archéologie, notamment lors de l'étude des inscriptions de la synagogue d'Hammam Lif, car ces textes avaient été fabriqués en mosaïque. De plus, des symboles accompagnaient souvent les inscriptions. Cette documentation était très dispersée, notamment dans les Jewish Symbo/s d'E.R. Goodenough ${ }^{22}$, qui étaient en outre incomplets, et dans les Lampes de

19 PietRi, Ch., «Un judéo-christianisme latin el l'Afrique chrétienne», dans Église et histoire du christianisme en Afrique (Congrès de Bologne, 1986), 1990, pages 1-12, propose de retirer du dossier plusierur textes qui son, en fait, des textes comportant simplement des éléments "judai"sants», et don je n'ai jamais dit qu'ils étaient «juifs» (voir le litre de l'article).

20 Solin, H., "Gli Ebrei d'Africa: una nota», dans l'Africa romana. Atti del VIII Convegno (1990), 1991, page 615,n. 1.

21 Vattioni, F., "Una iscrizione giudaica d Lepcis Magna. Ant. Afr.", XIX, 1983, 63-64, d'où A.E., $1983,950$.

22 Goodenough, E.R., Jewish Symbols, II, 1953, 63-68, 88, 92,95 et suiv., 109, III, 1953, n.․․ 865-873 (sauf 868), 886, 888, 890-906 (sauf 893), 913-921, 927, 938-940, 949-957, IV, 1954, 209. 
Carthage de J. Deneauve ${ }^{23}$. La publication des actes du congrès qui s'est tenu en 1983 à Montpellier a été l'occasion de proposer un court article rassemblant l'essentiel des documents archéologiques ${ }^{24}$, soit un unique plan de sinagogue dont l'identification soit incontestable, avec les mosaîques qui l'ornaient (il s'agit donc de la synagogue d'Hammam Lif, en Tunisie), deux nécropoles, à Tripoli et Carthage, plusieurs dizaines de lampes décorées du chandelier à sept branches, et quelques objets entrant dans la catégorie «Divers». A cette liste, j'ajouterai un luminaire à la menorah, inédit à ma connaissance et conservé dans une vitrine du musée d'Hippone. S. Dahmani, conservateur de ce site, m'a dit qu'elle avait été déposée là par $E$. Marec, et qu'il n'avait trouvé aucun renseignement sur son origine dans les maigres archives qu'il avait découvertes en prenant ses fonctions. Cette lampe peut provenir d'Hippone, ou une ancienne présence juive est prouvée par une des lettres de s. Augustin qui ont été récemment publiées ${ }^{25}$. Mais elle peut très bien avoir été apportée de n'importe où, d'Algérie, voire de Tunisie, et être venue là au titre d'échanges entre musées, pratique bien attestée au $X U X^{\ominus}$ et au début du $X X^{\ominus}$ siècle.

Dans ses recherches épigraphiques, P. Monceaux avait dû utiliser l'archéologie, mais aussi les sources littéraires. Quelques textes d'auteurs africains renvoient en effet à des événements précis. Par ailleurs, nous avons remarqués que chacun des Pères de l'Eglise originaire de ces provinces avait rédigé son Aduersus ludaeos ${ }^{26}$. La production de $\mathrm{s}$. Augustin en ce domaine avait d'ailleurs été particulièrement étudiée ${ }^{27}$. Et d'autres

23 Deneauve, J., Lampes de Carthage, 1969, n. ${ }^{\circ}$ 997, 1105-1107, 1113-1118.

24 LE BOHEC, Y., «Les sources archéologiques du jadaïsme africain sous l'Empire romain", dans Juifs et judaisme dans l'Afrique du nord dans l'Antiquité et le Haut Moyen-Age, sous la direction de $\mathrm{C}$. lancu et J.-M. Lassère, 1985, 13-47 (pl.).

25 DIVJAK, J., Epist., $8^{*}$ (voir 20*). Sur ce texte: ROUGÉ, J, «Escroquerie et brigandage en Afrique romaine au temps de $\mathrm{s}$. Augustin, (Epist., $8^{\star}$ et 20*), dans Les lettres de $S$. Augustin découvertes par J. Divjak. Communications présentées au colloque des 20 et 21 septembre 1982, 1983, pages. 177-188 et, en dernier lieu, CASTRITIUS, H., "The Jews in North Africa at the time of Augustine of Hippo. Their social and legal position", dans IXth World Congress of Jewish Studies, Div. B, vol. 1,1986, pages 31-37, article repris et développé dans Seid weder den Juden noch den Heiden noch de Gemeinde Göttes ein Ärgernis (1, Kor. 10, 32). «Zur sozialen und rechtlichen Stellung der Juden im Spätrömischen Nordafrika", dans Antisemitismus und jüdische Geschichte, Studien zu Ehren von Herbert A. Strauss, hrsg. v. R. Erb und M. Schmidt, 1987, pages 47-67.

26 LUKYN WILliams, A., Aduersus ludaeos, 1935, et Simon, M., Verus Israel. Études sur les relations entre Chrétiens et Juifs dans l'Empire Romain (135-425), 1964, 2e édit.

27 DouAls, G., «Saint Augustin et le judaisme», L'Université catholique, 1894, 5-25; BERARD, P. Saint Augustin et les Juifs, 1913, 87 pages.; Blumenkranz, B., Die Judenpredigt Augustins, 1945, et "Augustin ettes Juifs" Recherches augustiniennes, 1, 1958, 225-241, JoLY, R., "L'intolérance de s. Augustin, doctrine ou attitude?», dans Mél. M. Renard, I, 1969, 493-500 et RaveAux, Th., "Aduersus ludaeos. Antisemitismus bei Augustinus?", dans Signum pietatis. Festgabe für C.P. Mayer $=$ Cassiciacum, XL, 1989, 37-51. 
Pères en ont fait autant. Nous avons toutefois posé une question, sans d'ailleurs y répondre ${ }^{28}$. Étant bien entendu que ces critiques relèvent très largement du domaine théologique, et ne concernent pas toutes spécifiquement les Juifs d'Afrique, n'y a-t-il pas au moins quelques éléments oiriginaux, propres aux provinces qui nous occupent, dans ces traités? A. Lukyn Williams, lui, ne s'est pas posé la question. Un cas a cependant été privilégié, du point de vue qui nous occupe, c'est celui de Tertullien ${ }^{29}$. $\mathrm{Cl}$. Aziza s'est efforcé de reconstituer la vie quotidienne de la commauté de Carthage à partir des écrits de cet auteur (nous y reviendrons) ${ }^{30}$. De toute façon, on trouvera sans doute des références qui, dans d'autres auteurs, ont échappé a nos enquête il reste encore des recherches à faire. Et, parmi les lettres de s. Augustin publiées par J. Divjak, il en est une qui mentionne un Juif, riche propriétaire esclavagiste de la région d'Hippone ${ }^{31}$.

On pouvait croire qu'avec les inscriptions, l'archéologie et les Pères de l'Église, on avait fait le tour des sources disponibles, à condition d'y ajouter quelques références prises au Talmud de Jérusalem. Or, au congrès de Montpellier qui a déjà été évoqué, A. Linder révélait qu'en cherchant dans les recueils de lois, le Code Théodosien et le Code Justinien, il avait trouvé treize textes qui se rapportaient directement à notre sujet, et cent sept qui le touchaient de plus ou moins prés ${ }^{32}$. II remarquait que, pour l'essentiel, le pouvoir impérial se souciait de limiter le prosélytisme et d'empêcher les Juifs de s'allier au païens et aux hérétiques contre les chrétiens orthodoxes soutenus par le pouvoir impérial. Une législation aussi abondante présentait deux centres d'intérêt. Elle prouvait l'importance des Juifs en Afrique au Bas-Empire, et elle, montrait qu'ils ne craignaient pas de chercher à faire des recrues, ce qui est passablement original. Et $\mathrm{A}$. Linder est revenu sur ces questions dans un livre très récent ${ }^{33}$.

28 LE BOHEC, S. et $Y$., "Juifs et chrétiens de la Judée à l'Afrique», dans L'histoire des croyants, Mémoire vivante des hommes. Mél. Ch. Molette, 1989, page 572.

29 Outre Cl. AZIZA, cité note suivante, on verra, en dernier lieu: HoRBURY, W., «Messianism among Jews and Christians in the second century", Augustinianum, XXVIII, 1988, pages 71-88, et LenNan, R. S. Mac, Four early Christian Texts on Jews and Judaism in the 2nd c. Chr. E., Diss. University of Minnesota, Minneapolis, 1988 page, $368=D A, L, 1989,520 A-521 \mathrm{~A}$.

30 AZIZA, Cl., Tertullien et le judaïsme, 1977, V-327, et "Quelques aspects de la communauté juive de Carthage au $\|^{\mathrm{e}}$ siècle d'après Tertullien", REJ, CXXXVII, 1978, page 491 et suivantes.

31 Epist., $8^{*}$ (voir plus haut références a J. Rougé et $H$. Castritius).

32 LINDER, A., "La loi romaine et les Juifs d'Afrique du nord", dans Juifs et judaïsme dans l'Afrique du nord dans l'Antiquité et le Haut Moyen-Age, sous la direction de C. lancu et J.-M. Lassère, 1985, pages 57-64.

33 Voir, du même, The Jews in Roman Imperial Legislation, 1987, page 436. 
On n'a pas attendu l'achèvement de cette compilation des documents pour proposer des essais de synthèse. Élève de M. Le Glay, J.-M. Lassère faisait une large part au judaïsme africain dans son ouvrage magistral consacré au peuplement de l'Afrique romaine et intitulé Vbique populus ${ }^{34}$. Après un examen critique des légendes concernants les origines ${ }^{35}$, il présentait les sources ${ }^{36}$ et établissait une liste d'individus ${ }^{37}$. Son travail l'amenait à proposer deux thèses. Une étude onomastique ${ }^{38}$ concluait à une "origine probablement italienne» "du judaïsme maghrébin ${ }^{39}$; par ailleurs, il pensait que l'existence d'un judaïsme berbère n'était pas prouvée pour l'Antiquité ${ }^{40}$. Poursuivant, de son côté, une vaste enquête sur les Juifs et les Syriens dans le monde romain, $\mathrm{H}$. Solin publiait en premier lieu une courte notice, un article de revue ${ }^{41}$, en second lieu une étude plus détaillée, en deux parties, présentant et discutant d'abord les sources, ensuite les interprétations des historiens ${ }^{42}$, et enfin une brève «note» ${ }^{43}$. Le second travail mentionné, avec toute son érudition, nous dispense de citer ici l'ensemble de la bibliographie; pour cette dernière, nous y renvoyons donc. On ajoutera a ce tableau une courte notice de J.-M. Lassère sur un site particulier, le locus ludaeorum Augusti ${ }^{44}$, et une série de réflexions sur l'onomastique des Juifs qui ont vécu en Afrique romaine ${ }^{45}$. L'onomastique suggère plusiurs coriclusions. Parmi les Juifs qui ont vécu dans l'Afrique romaine, les humbles représentaient plus des $2 / 3$ des gens connus. Les riches, qui ont d'abord vécu dans les cités du littoral, se sont ensuite dispersés dans l'intérieur du pays. La latinisation de cette population, moindre qu'on n'a dit, n'était puissante qu'en Numidie et en Césarienne ${ }^{46}$.

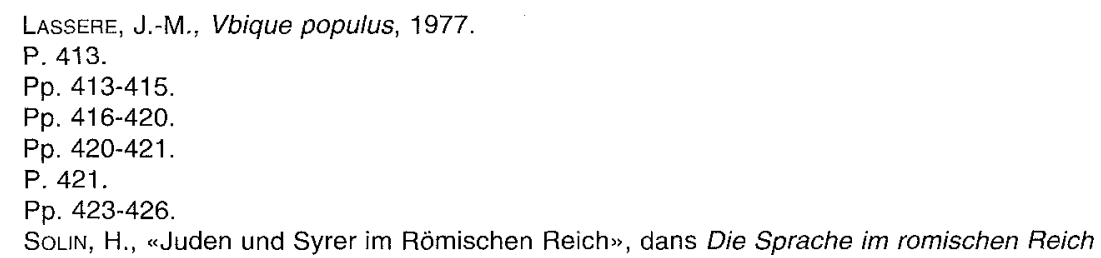

SOLIN, H., "Juden und Syrer im Römischen Reich", dans Die Sprache im romischen Reich der Kaiserzeit, BJ. Beihefte, XL, 1980, pages 326-327.

42 SoLIN, H., «Juden und Syrer im westlischen Teil der romïschen Welt», dans ANRW, II, 29, 2, 1983 , pages $770-779$.

${ }_{43}$ SolIN, H., "Gli Ebrei d'Africa: una nota», dans L'Africa romana. Atti del VIII Convegno (1990), 1991, pages 615-623.

44 LASSERE, J.-M., "Locus ludaeorum Augusti: une hypothèse", dans Juifs et judaïsme dans l'Afrique du nord dans l'Antiquité et le Haut Moyen-Age, sous la direction de C. lancu et J.-M. Lassère, 1985, pages 65-72.

45 LE BOHEC, $Y$., «Juifs et judaïsants dans l'Afrique romaine: remarques onomastiques»., Ant. Afr. XVII, 1981, pages 209-229.

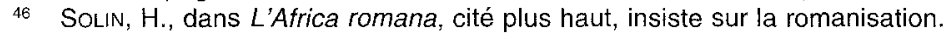


Revenons à la grosse étude d'H. Solin, car elle permet en outre une intéressant conclusion, tirée d'un parallèle à faire entre ce que l'on peut observer en Afrique d'une part, en Gaule et en Espagne d'autre part ${ }^{47}$; on en conclura que la présence juive a été plus forte au Maghreb que dans les autres provinces de l'Occident romain, et qu'elle y a été relativement tardive, puisqu'elle n'est bien attestée qu'au Bas Empire ${ }^{48}$.

Pour les études locales ou régionales, deux séries retiendront particulièrement l'attention. D'une part, c'est Carthage qui a été l'objet des soins d'abord de G. Otranto ${ }^{49}$, puis surtout de Cl. Aziza ${ }^{50}$, éminent spécialiste de Tertullien, auxquels on ajoutera une utile référence a W. H. C. Frends 51. D'autre part la Maurétanie Tingitane, à l'autre extrémité du Maghreb, a suscité d'importantes recherches. $\mathrm{O}$. Giordano ${ }^{52}$ avait soutenu la thèse que le christianisme était venu dans cette province en suivant les voies du judaïsme, qu'il distinguait des voies de la romanisation, à tort a notre avis: Romains, paiens, juifs et chrétiens ne disposaient que d'un seul réseau de routes ou d'une seule ligne de navigation. Par la suite, E. Frézouls publiait une importante inscription qui prouvait l'existence d'une synagogue à Volubilis ${ }^{53}$ et, la meme année, $M$. Euzennat donnait une étude magistrale sur les Orientaux qui avaient vécu dans cette province ${ }^{54}$ (pour notre propos, donc, une communauté à Volubilis, plus un individu à Chellah). Plus tard, venait un autre travail d'E. Gozalbes Cravioto ${ }^{55}$.

On ne négligera pas une ultime synthèse, due à C. Gebbia ${ }^{56}$, qui approuve une chronologie tardive pour l'apparition du judaïsme au Maghreb. Elle montre également comment les Juifs s'inséraient dans le milieu économique et culturel du Ive siècle, une période qu'elle connaît particulièrement bien.

47 Solin, H., dans ANRW, II, 29, 2,1983, pages 749-760.

48 PICARD, G.-Ch., La civilisation de l'Afrique romaine, 1990, $2^{e}$ édit., page 151.

49 Otranto, G., Giudei e cristiani a Cartagine tra ll e llis. L'Aduersus ludaeos di Tertulliano, 1 L 1975 , page 172.

50 AzIZA, Cl., cité plus haut.

51 Frend W. H. C., "Jews and Christians in third century Carthage», dans Mél. M. Simon, 1978, pages 185-194.

52 Giordano, O., «La Mauretania Tingitana e il cristinesimo primitivo», Nuovo Didaskaleion, $\mathrm{XV}, 1965$, pages 25-51.

53 FrÉzouls, E., «Une synagogue juive attestée à Volubilis», dans Acta of the 5th International Congress of Greek and Latin Epigraphy (Camoridge, 1967), 1971, pages 287-292.

54 Euzennat, M., «Juifs et Orientaux en Maurétanie Tingitane», Ant. Afr., V, 1971, pages 161-178.

55 Gozalbes Gravioto, E., "Los Judios en Mauretania Tingitana», Studi Magrebini, XI, 1979 133-166, et «El cristianismo en Mauritania Tingitana», Cuadernos de la Biblioteca española de Tetuan, 23-24 juin-dec. 1981, pages 279-309 (non uidi).

56 GEBBIA, C., "Le comunità giudaiche nell'Africa romana antica e tardo-antica», dans L'Africa romana, II, 1986, pages 101-111. 
Malgré sa relative abondance, la documentation concernant le judaïsme dans l'Afrique romaine impose des limites aux synthèses qui ont été tentées, on l'a dit. Certaines difficultés paraissent assez évidentes pour que l'accord se soit fait à leur propos. D'autres, au contraire, ont donnée matière a débat. Et ce n'est pas moins de six problèmes différents qui peuvent etre énumérés.

En premier lieu, il convient d'apprécier avec précision la portée des textes littéraires. Cl. Aziza, on l'a dit, a décrit la vie quotidienne des Juifs de Carthage à partir des écrits de Tertullien ${ }^{57}$. Les oeuvres de ce Père de l'Eglise contiennent effectivement de nombreuses références intéressantes. Certaines concernent, on l'a dit, les Juifs d'Afrique du nord. Mais d'autres ont une portée plus générale, et concernent les Juifs de l'ensemble de l'empire romain, ce qui d'ailleurs ne signifie pas qu'elles ne s'appliquent pas à la communauté objet de notre étude. II nous semble toutefois qu'il faut être prudent avec cet auteur. Dans un article sous presses, nous soutenons la thèse que l'anecdote rapportée au début du De corona, qui intéresse l'histoire de l'armée et pas celle du judaïsme, n'a eu pour cadre ni Carthage ni Lambèse, contrairement à ce qu'a écrit l'historiographie antérieure; c'est à Rome qu'elle a eu lieu ${ }^{58}$. Cette petite constatation entraîne une grande conséquence: c'est l'ensemble des Pères de l'Église africains qu'il faut aborder avec prudence. Malgré l'absence de journaux, de radio et de télévision, des informations en assez grand nombre pouvaient être largement diffusées.

Sur un deuxième point, le conflit est net, et il n'est pas tranché. II s'agit de l'origine des Juifs qui ont vécu dans l'Afrique romaine: venaient-ils directement de Palestine, ou ont-ils fait un détour par l'Italie en général, et par Rome en particulier? Si l'auteur de la présente communication ${ }^{59}$, ainsi que, notamment, G. Quispel ${ }^{60}$ et $\mathrm{H}$. Solin ${ }^{61}$, penche pour la première hypothèse, J.-M. Lassère incline plutôt vers la seconde ${ }^{62}$. II faut bien reconnaître que nous ne disposons que de peu d'indices explicites, et la seule étude de l'onomastique n'apporte pas de preuve indiscutable. Et on voit mal comment proposer une réponse définitive à cette question en l'absence de nouvelles découvertes.

\footnotetext{
57 AZIZA, Cl., cité plus haut.

58 LE BOHEC, Y., «Tertullien, De corona, 1: Carthage ou Lambèse?», REAug, à paraître.

59 LE BOHEc, Y., Ant. Afr., XVII, 1981, page 229.

60 Quispel, G., «African Christianity before Minucius Felix», dans Mél. H. L. N. Nelson, édités par J. Den Boft et A. H. M. Kessels, 1982, pages 257-335.

${ }_{61}$ Solın, $H_{\text {, }}$ dans L'Africa romana, cité.

62 LASSËRE, J. M., Vbique populus, page 421.
} 
On abordera, en troisième lieu, un débat plus brûlant, celui des relations entre Juifs en chrétiens ${ }^{63}$. Ici, on utilisera d'abord une étude d'ensemble très récente ${ }^{64}$. En outre, deux domaines ont été particulierement bien étudiés, Carthage et la Maurétanie Tingitane. A vrai dire, à ce propos, on ne peut pas se limiter au seul cas de l'Afrique romaine. Beaucoup de savants, et non des moindres, ont soutenu ce que l'on pourrait appeler la «thèse des bons rapports» ${ }^{65}$. Ils expliquent que les premiers chrétiens ont préché dans des synagogues, ont enterré leurs morts dans des nécropoles juives, et qu'il ne pouvait pas y avoir de conflits entre monothéistes adorateurs du même Dieu. lis relèvent un argument-conséquence, de poids à leurs yeux: là où on trouve des communautés juives, on trouve des communautés chrétiennes. 0 . Giordano ${ }^{66}$, qui a été déja cité, a soutenu que le christianisme était juif pendant tout le $1^{\text {er }}$ siecle et que, pour atteindre la Maurétanie Tingitane, il avait suivi les voies du judaïsme et non celles de la romanisation. E. Gozalbes Cravioto ${ }^{67}$ va dans le même sens, avec une nuance: il faut également prendre en compte les Orientaux, donc les voies commerciales.

Cette «thèse des bons rapports» a toutefois été contestée. En 1971, T. D. Barnes, a propos de Carthage ${ }^{68}$, et E. Frézouls, a propos de la Maurétanie Tingitane ${ }^{69}$, arrivaient aux mêmes conclusions. Le premier pensait que le sentiment dominant entre les deux communautés était la haine ${ }^{70}$, que c'étaient des marchands orientaux et non des Juifs qui avaient introduit la nouvelle religion a Carthage ${ }^{71}$, et qu'il n'y avait pas eu de contacts étroits entre Tertullien et les Juifs ${ }^{72}$, point sur lequel il a été

63 SIMON, M., Verus /srael. Études sur les relations entre Chrétiens et Juifs dans l'empire romain (135-425), 1964, $2^{\ominus}$ édit; LEANEY, A. B. C., The Jewish and the Christian World 200 B.C. to A.D. 200, 1984, page 279. (Cambridge Commentary on the Writing, VII).

64 Pietri, Ch., article cité.

65 Otranto, G., et AzIZA, Cl., cités plus haut, par exemple, et surtout ce dernier, «Ouelques aspects de la polémique judéo-chrétienne dans l'Afrique romaine (ile-V|e siècles)", dans Juifs et judaïsme dans l'Afrique du nord dans l'Antiquité et le Haut Moyen-Age, sous la direction de C. Iancu et J.-M. Lassère, 1985, page 50: «De bons rapports entre les deux communautés»,. Voir $S$. et $Y$. Le Bohec, cités plus haut. Mais aussi Ch. Pietri, article cité, qui propose une vision très radicale de ces rapports: il parle en effet d'une «dialectique d'échanges et de conflits», mais semble voir plus d'échanges que de conflits, là où nous voyons plus de conflits que d'échanges.

66 Giordano, O., cité plus haut.

67 Gozalbes Gravioto, E., cité plus haut.

68 BARNES, T. D, Tertullian. A historical and literary Study, 1971, pág. 90, contesté par BRAUN R., REL., L, 1972, 84.

69 Frezouls, E., cité plus haut.

70 Barnes, T. D., ouvrage cité, pages 90-93.

71 BARNES, T. D., ouvrage cité, page 64.

72 BaRnes, T. D., ouvrage cité, page 92. 
contesté, entre autres d'ailleurs par $\mathrm{R}$. Braun ${ }^{73}$. Le second allait même au-delà: le conflit était si aigu, si vif, que le christianisme éprouvait du mal à se diffuser là où existaient des communautés juives.

Récemment, moi-même et mon épouse nous sommes rangés sans hésitation dans le camp de T. D. Barnes et d'E. Frézouls ${ }^{74}$ : en examinant la documentation africaine, nous n'avons pas trouvés de chrétiens dans les synagogues, ni dans les nécropoles talmudiques, ni, d'un maniere générale, là où se trouvaient des Juifs. On pourrait ajouter deux arguments. D'abord, comme l'a montré M. Sotomayor pour le cas de l'Espagne ${ }^{75}$, la diffusion d'une religion peut se faire par plusieurs canaux, et la péninsule ibérique a ainsi été évangélisée par des Africains, mais aussi par des Romains et des Orientaux. Ensuite, les études qui ont été consacrées au judaïsme dans les provinces de l'Occident romain en général, de l'Afrique en particulier, font penser que le christianisme est arrivé dans ces régions en même temps que le judaïsme, et peut-être même avant lui ${ }^{76}$.

La «thèse des bons rapports", qui nous paraît erronée, s'explique peutêtre par de bons sentiments, le souci de certains chrétiens qui, à l'heure actuelle, veulent manifester leur sympathie à un peuple envers lequel leurs prédécesseurs ont été parfois injustes. Mais les bons sentiments font de la mauvaise histoire. Cette théorie repose surtout sur une double erreur de perspective. Il est en effet incontestable que le christianisme puise une partie de sa doctrine dans la Bible, mais il ne reconnaît pas le Talmud, pas plus que le judaïsme n'admet le Nouveau Testament. Les missionnaires chrétiens, de plus, suivaient les mêmes voies que les émigrants juifs, et les grands centres attiraient les uns et les autres. Les religions, orientales ou autres, suivaient les routes romaines, parce qu'il n'y en avait pas d'autres. Il conviendra toutefois à l'avenir de ne pas tomber dans l'excès inverse: si conflits il y a eu, ces conflits n'étaient ni permanents ni généralisés .

Nous arrivons ensuite à des problèmes qui soulèvent moins les passions, mais qui sont plus difficiles a résoudre, en raison des lacunes de la documentation. On doit en effet se demander, en quatrième lieu, à quel milieu socio-économique, au singulier ou au pluriel, appartenaient les Juifs

\footnotetext{
73 Braun, R., REL, L, 1972, page 84.

74 Le BOHEc, S. et Y., article cité dans Mél. Ch. Molette.

75 Sotomayon, M., "Reflexión histórico-arqueológica sobre el supuesto origen atricano del cristianismo hispano", dans IX Symposium de prehistoria i arqueologia peninsular, "I reunió d'arqueologia paleocristiana hispanica (Montserrat, 2-5 nov. 1978), Institut d'arqueologia i prehistoria, publication eventuals, XXXI 1982, pages 11-29.

76 Solin, H., dans ANRW, II, 29, 2, 1983, pages 749-760.
} 
des provinces africaines. L'examen des sépultures de Carthage, notamment, et de la synagogue d'Hammam Lif, l'étude de l'onomastique et de la dispersion géographique des personnes concernées, ont permis de formuler quelques hypothèses: l'immense majorité de ces gens vivait dans la pauvreté, mais une minorité devait se rapprocher du milieu des notables municipaux ${ }^{77}$. Bien sur, l'image du banquier juif relève de l'anachronisme pur et simple. On peut supposer que beaucoup vivaient des petits métiers communs à tous les ports de l'Antiquité. Quelques-uns devaient travailler la terre ou en posséder. C'est ce que confirme une des lettres de S. Augustin récemment publiées par J. Divjak ${ }^{78}$ : elle met en scène un Juif du nom de Licinius qui exploitait une propriété de type esclavagiste. Les Juifs d'Hippone ont d'ailleurs retenu l'attention de Th. Raveaux ${ }^{79}$ : les remarques précises et justes de $s$. Augustin sur le sabbat s'expliquent a ses yeux par une observation directe de cette communauté. II ne faut cependant pas oublier que l'évêque d'Hippone avait voyagé, qu'il connaissait Carthage et l'Italie, et avait pu puiser ailleurs ses informations.

Cette question de l'observance du sabbat amène a un cinquième point, celui de l'attitude religieuse des Juifs africains. Tout ce que l'on peut dire, c'est que ces derniers semblent s'être comportés comme tous leurs compatriotes de la Diaspora après la prise de Jérusalem par Titus, c'est-à-dire qu'ils on suivi le pharisaïsme ${ }^{80}$. Rien ne les distingue, rien ne permet d'imaginer une quelconque attitude hétérodoxe: la synagogue d'Hammam Lif et la nécropole de Gamarth ne présentent aucun caractère particulier, ressemblent à ce que l'on voit ailleurs, et suivent les prescriptions du Talmud, qui d'ailleurs mentionne quelques rabbins carthaginois. Et les Pères de l'Église africains ne signalent eux non plus rien d'extraordinaire. Nous verrons cependant que le judaïsme africain a débordé le cadre de la nationreligion, et nous devons letre attentifs au fait qu'ici nous utilisons surtout l'argument a silentio. Donc, prudence.

77 Le BOHEC, Y., Ant. Afr., XVII, 1981, pages 169-170, et dans Juifs et judaïsme dans l'Afrique du nord dans I' Antiquité et le Haut Moyen-Age, sous la direction de C. lancu et J.-M. Lassère, 1985,24 . Point de vue partagé par SoliN, H., dans L'Africa romana, cité plus haut.

${ }_{78}$ DivjaK, J., Epist., $8^{*}$ (voir 20*). Castritius, H., «Seid weder den Juden noch den Heiden noch der Gemeinde Göttes ein Ärgernis (1, Kor. 10. 32). Zur sozialen und rechtlichen Stellung der Juden im Spätrömischen Nordafrika", dans Antisemitismus und jüdische Geschichte, Studien zu Ehren von Herbert A. Strauss, hrsg. v. R. Erb und M. Schmidt, 1987, pages 47-67. Voir Th Raveaux, note suivante.

79 RaveauX, Th., «Augustinus üben den jüdischen Sabbat der seiner Zeit», REAug, XXVIII, 1982, pages 213-224.

80 LE BOHEC, Y., Ant. Afr., XVII, 1981, pages 170-171, et dans Juifs et judaisme dans l'Afrique du nord, 1985, page 24. 
II faudrait aussi beaucoup de prudence pour aborder le sixième problème soulevé par le sujet que nous traitons. II s'agit précisément de la diffusion du judaïsme au sein des populations africaines ${ }^{81}$, païennes et chrétiennes. II convient de distinguer deux processus. D'une part, certains savants ont admis que le prosélytisme juif avait connu le succès auprès des indigènes. Récemment, G. Camps a émis l'hypothèse que les Juifs des régions situées au nord du Sahara auraient été convertis sous l'Empire romain à la suite de contacts établis a partir de la Cyrénaïque et de la Tripolitaine; quant à ceux qui sont attestés dans la partie occidentale de cette zone, ils auraient été judaïsés par les nomades chameliers zénètes, mais pas avant le ve siècle ${ }^{82}$. II déclare cependant clairement qu'il ne s'agit que d'hypothèses. Les partisans de cette théorie fondent leur argumentation sur une phrase d'Ibn Khaldoun qui a écrit que' «une partie des Berbères professait le judaïsme" ${ }^{83}$. Cet unique argument a rencontré un certain scepticisme ${ }^{84}$, pour ne pas dire plus ${ }^{85}$. En l'absence de preuves archéologiques, il nous paraît qu'il convient, pour l'instant, de se montrer réservé. Par ailleurs, la présence juive a donné naissance a des syncrétismes. Des individus ont ajouté à leurs croyances ou à leurs pratiques des élements empruntés au judaïsme et non admis par l'orthodoxie chrétienne. Le fidèle qui "craignait (Dieu)», en fait le Dieu des Juifs, se faisait appeler metuens ${ }^{86}$. Des sectes mélangeaient également des éléments d'origines diverses. Tels étaient les caelicoles ${ }^{87}$, auxquels un travail relativement récent a attribué une lampe décorée du chandelier a sept branches ${ }^{88}$, attribution qui ne nous paraît pas parfaitement établie; les caelicoles adoraient Caelestis-Tanit, d'où leur nom, mais recevaient le baptême et se donnaient des «noms juifs», ce qui les faisait passer pour tels. Les abéloniens ${ }^{89}$, également, se situaient aux confins des trois religions. Ils

81 PIETRI, Ch., "Un judéo-christianisme latin et l'Afrique chrétienne», dans Église et histoire du christianisme en Afrique (Congres de Bologne, 1986), 1990, pages 1-12.

82 CAMPS, G., "Réflexions sur l'origine des Juifs des régions nord-sahariennes", dans Communautés juives des marges sahariennes du Maghreb, 1982, pages 57-67.

83 SIMON, M., "Le judaïsme berbère dans l'Afrique ancienne», RHPhR, XXVI, 1946, pages 131 et 105-145 (repris dans Recherches d'histoire judéo-chrétienne, 1962).

84 LASsére, J.-M., Vbique populus, 1977, pages 423-426; SOLIN, H., dans ANRW, II, 29, 2, page 777.

85 FANTAR, M. H., "La Kahina, reine des Berbères", Reppal, Ill, 1987, pages 169-184, en particulier 176-178 («La religion de La Kahina »), ou il la présente comme une païenne.

86 LE BOHEC, Y., Ant. Afr., XVII, 1981, n. ${ }^{\circ} 72$.

87 SoLIN, H., dans ANRW, II, 29, 2, 774.

88 SIMON, M., «Un document du syncrétisme religieux dans l'Afrique romaine», CRAl, 1978, pages 500-524.

${ }_{89}$ SIMON, M., «Ill. Le syncrétisme judéo-chrétien», dans «Le judaïsme berbère dans l'Afrique ancienne", $R H P h R, 1946$, pages 1 et suiv., 101 et suiv. 
prétendaient se rattacher à Abel, le pur, celui qui n'a pas procréé, pour recommander la chasteté.

Ces difficultés ne découragent cependant pas ceux qui ont entrepris des recherches dans un domaine aussi passionnant. Deux projets sont ainsi en cours d'élaboration. C. Gebbia prépare une publication des textes non épigraphiques (le Talmud, les Codes de lois et les auteurs chrétiens); on pourra ainsi disposer de l'ensemble des sources. Et moi-même j'envisage un ouvrage sur les Racines juives au Maghreib; il faudra admettre que certains pans de la réalité nous échappent, en raison des lacunes de la documentation; mais, étant donné ce qu'est l'orientation actuelle de la recherche archéologique et épigraphique en Afrique du nord, on peut dif-ficilement s'attendre, à propos du judaïsme, a d'importantes découvertes dans les années à venir. D'où la possibilité, et la nécessité, de cette synthese .

\section{PUBLICATIONS CONCERNANT LE JUDAÏSME AFRICAIN PARUES DE- PUIS 1977}

En choisissant de commencer cette bibliographie à l'année 1977, nous souhaitons attirer l'attention sur l'importance du travail de J.-M. Lassère en ce domaine. La bibliographie ancienne se trouve rassemblée dans nos propres travaux cités ci-dessous, et dans l'article fondamental a cet égard de H. Solin, également mentionné plus loin.

AzIZA, Cl., Tertullien et le judaïsme (1977), v, page 327.

- "Quelques aspects de la communauté juive de Carthage au $\|^{e}$ siècle d' après Tertullien", REJ CXXXVII (1978), pages 491 et suiv.

- «Quelques aspects de la polémique judéo-chrétienne dans l'Afrique ramaine ( $\mid]^{0}-\mathrm{V}^{\mathrm{a}}$ siècles)", dans Juifs et judaïsme dans I"Afrique du Nord dans I'Antiquité et le Haut MoyenAge, sous la direction de C. Lancu et J.-M. LASSĖRE, 1985, pages 49-56.

CAmps, G., "Réflexions sur l' origine des Juifs des régions nord-sahariennes", dans Communautés juives des marges sahariennes du Maghreb. 1982, pages 57-67.

CAstrittus, $H_{\text {., }}$ "The Jews in Norih Africa at the time of Augustine of Hippo. Their social and legal position", dans IXth World Congress of Jewish Studies, Div. B., vol. I. 1986, pages 31-37.

- "Seid weder den Juden noch den Heiden noch der Gemeinde Göttes ein Argernis (1, Kor. 10, 32).Zur sozialen und rechtlichen Stellung der Juden im Spätrömischen Nordafrika», dans Antisemitismus und Jüdische Geschichte, Studien zur Ehren von Herbert. A. Strauss, hrsg. v. R. Erb und M. Schmidt. 1987, pages 47-67.

FANTAR, M.H., "La Kahina, reine des Berbères", Reppal III (1987), pages 169-184, en particulier 176-178 («La religion de La Kahina»).

FREND, W.H.C., "Jews and Christians in third century Carthage», dans Mélanges M. Simon. 1978, pages 185-194.

GEBBIA, C., "Le comunità giudaiche nell'Africa romana antica e tardo-antica», dans L'Africa romana II (1986), pages 101-111.

Gozalbes Cravioto, E., "Los Judíos en Mauretania Tingitana", Studi Magrebini XI (1979), 133-166.

- "El cristianismo en Mauritania Tingitana", Cuadernos de la Biblioteca española de Tetuán. 23-24 juin-dec. 1981, pages 279-309. 
Horbury, W., «Messianism among Jews and Christians in the second century», Augustinianum XXVIII (1988), pages 71-88.

LASSĖRE, J.-M., Vbique populus. 1977, pages 413-426.

- "Locus ludaeorum Augusti: une hypothèse", dans Juifs et judaïsme dans l'Afrique du nord dans l' Antiquité et le Haut Moyen-Age, sous la direction de C. lancu et J.-M. Lassère. 1985, pages 65-67.

LE BOHEC, Y., «Inscriptions juives et judaïsantes de l'Afrique romaine», Ant. Afr. XVII (1981), pages $165-207$.

- "Juifs et judaïsants dans l'Afrique romaine. Remarques onomastiques", ibidem, pages 209229.

- "Les sources archéologiques du judaïsme africain», dans Juifs et judaïsme dans l'Afrique du nord dans l'Antiqueté et le Haut Moyen-Age, sou la direction de C. Lancu et J.-M. Lassère. 1985, pages 13-47.

- En collaboration avec LE BOHEC, S., «Juifs et chrétiens de la Judée à l'Afrique», dans L'histoire des croyants, Mémoire vivante des hommes, Mél. Ch. Molette. 1989, pages 567575.

LINDER, A., "La roi romaine et les Juifs d'Afrique du nord", dans Juifs et judaïsme dans l'Afrique du nord dans l'Antiquité et le Haut Moyen-Age, sous la direction de C. Lancu et J.-M. Lassère, 1985, pages 57-64.

- The Jews in Roman Imperial Legislation. 1987, page 436.

LENNAN, R.S. Mac, four early Christian Texts on Jews and Judaism in the IInd c. Chr. E., Diss. University of Minnesota, Minneapolis, 1988, page $368=D A L$ (1989), 520A-521A.

PIETRI, Ch., «Un judéo-christianisme latin et l'Afrique chrétienne», dans Église et histoire du christianisme en Afrique (Congrés de Bologne, 1986). 1990, pages 1-12.

QuiSPEL, G., "African Christianity before Minucius Felix", dans Mél. H.L.N. Nelson, édités par J. Den Boft et A.H.M. Kessels. 1982, pages 257-335.

RavEAUX, Th., "Augustinus über den jüdischen Sabbat der seiner Zeit», R.E.Aug. XXVIII (1982), pages 213-224.

- "Aduersus ludaeos. Antisemitismus bei Augustinus?», dans Signum pietatis. Festgabe für $C$. P. Mayer = Cassiciacum XL (1989), pages 37-51.

RovGÉ, J., "Escroquerie et brigandage en Afrique romaine au temps de s. Augustin (Eist., $8^{\star}$ et $20^{*}$ )", dans Les lettres de s. Augustin découvertes par J. Divjak, Communications présentées au colloque des 20 et 21 septembre 1982, 1983, pages 177-188.

SimON, M., "Un document du syncrétisme religieux dans l'Afrique romaine», CRAI. 1978, pages 500-524.

SoLIN, H., "Juden und Syrer im römischen Reich», dans Die Sprache im römischen Reich der kaiserzeit, B.J., Beihefte XL (1980), pages 326-327.

- «Juden und Syrer im westlischen Teil der römischen Welt», dans ANRW II, 29, 2 (1983), pages $770-779$.

- "Gli Ebrei d'Africa: una nota», dans L'Africa romana, Atti del VIII Convegno (1990), 1991, pages 615-623.

VATTIONI, F., "Una iscrizione giudaica di Lepcis Magna», Ant. Afr. XIX (1983), pages 63-64 (d' où $A E, 1983,950)$. 


\section{Anexo: \\ Ciclo "guerra y religión en el mundo antiguo»}


